\title{
THE EM RESPONSE DUE TO A PLANE SHEET OF ARBITRARY SHAPE AND CONDUCTIVITY PROFILE
}

\author{
D. G. HURLEY ${ }^{1}$ and P. F. SIEW ${ }^{2}$
}

(Received 18 October 1993; revised 23 February 1994)

\begin{abstract}
The eddy currents induced in a thin sheet of variable conductivity by a sinusoidally varying primary magnetic field are investigated in the low frequency limit when the depth of penetration of the primary field is much greater than the thickness of the sheet. The problem is formulated in terms of a set of integro-differential equations. The method of solution is applicable to bodies with arbitrary planar shape and the result is particularly useful in inverse problems involving bodies with conductivity inhomogeneities.
\end{abstract}

\section{Introduction}

The electromagnetic response of a thin conducting sheet in the low-frequency limit when the depth of penetration of the primary magnetic field is much greater than the thickness of the sheet is of interest in a number of branches of geophysics including atmospheric physics and geophysical exploration.

Maxwell [8] solved the problem for a uniform sheet of infinite extent. Extensions to uniform sheets of finite extent are given in $[1,3,6,10,11]$ and to nonuniform sheets of infinite extent in Price [9], and Ashour and Price [2]. In all these treatments the eddy-current density is assumed to be constant across the thickness of the sheet.

In the present paper, a perturbation scheme is set up in terms of a small parameter, $\delta$, which is the ratio of the thickness of the sheet to its lateral extent. The governing equations for the zero and first order potentials due to the induced currents in a sheet with variable conductivity are obtained. The zero order equations agree with that derived by Price [9]. The first order solution will, in general exhibit variations in the eddy-current density across the sheet [4]. It is shown that the problem can be recast in terms of an integro-differential equation which can be solved successively for the

\footnotetext{
'University of Western Australia, Nedlands, WA 6009.

${ }^{2}$ School of Mathematics and Statistics, Curtin University of Technology, WA.

(C) Australian Mathematical Society, 1995, Serial-fee code 0334-2700/95
} 
zero and first order solutions. Some numerical examples are given.

\section{Basic equations}

Consider a thin plane sheet of material of thickness $2 d$, and let $O x y z$ be a set of rectangular axes with $O x y$ lying in the plane of symmetry of the sheet. The sheet of conductivity $\sigma(x, y)$ is assumed to be surrounded by vacuum in which there is a sinusoidally varying primary magnetic field $\mathbf{H}^{(p)} \exp (i \omega t)$. If $\mathbf{H}$ is the total magnetic field with corresponding induction $\mathbf{B}$, then, neglecting displacement currents, the governing equations outside the sheet are

$$
\nabla \cdot \mathbf{B}=0 \quad \text { and } \quad \nabla \times \mathbf{H}=0
$$

where $\mathbf{H}=\mathbf{H}^{(p)}+\mathbf{H}^{(s)}$ is composed of the primary field and a secondary field $\mathbf{H}^{(s)}$ due to currents induced in the conductor, and a time variation $\exp (i \omega t)$ is assumed throughout. Inside the sheet the corresponding equations are

$$
\nabla \times \mathbf{E}=-i \omega \mathbf{B}, \quad \nabla \cdot \mathbf{B}=0, \quad \nabla \times \mathbf{H}=\sigma \mathbf{E},
$$

where $\mathbf{E}$ is the electric field. Taking the curl of the last of (2.2), gives

$$
\nabla^{2} \mathbf{H}+\nabla \sigma \times \mathbf{E}=i \mu \sigma \omega \mathbf{H}
$$

where $\mathbf{H}=\left[H_{x}, H_{y}, H_{z}\right]$.

Equations (2.1) imply the the existence of a scalar potential $\Phi$ such that

$$
\mathbf{H}^{(s)}=-\nabla \Phi, \quad \nabla^{2} \Phi=0 .
$$

We assume that the lateral extent of the sheet and the length scales for variations in its conductivity and variations in the amplitude of $\mathbf{H}^{(p)}$ are each of order $L$ and introduce the small parameter

$$
\delta=d / L .
$$

The analysis from this point on is similar to that reported in Hurley and Siew [4], which we will refer to as Problem I. One difference from Problem I is that now the conductivity is a function of $x$ and $y$, and using $\Sigma$ as a representative value of $\sigma$, we now define the induction number as

$$
\alpha=\mu \Sigma \omega d L .
$$

Proceeding as in Section 3 of Problem I, we first consider the inner region which is the interior of the sheet. Here we use the variables

$$
x^{\prime}=x / L, \quad y^{\prime}=y / L, \quad z^{\prime}=z / d, \quad \mathbf{h}^{*}=\mathbf{H}^{(s)} / \mathscr{H}, \quad \sigma^{*}=\sigma / \Sigma,
$$


where $\mathscr{H}$ is a representative value of the primary field. Dropping the asterisks we assume

$$
\mathbf{h}=\mathbf{h}^{(0)}+\delta \mathbf{h}^{(1)}+\delta^{2} \mathbf{h}^{(2)}+\cdots
$$

and find that instead of (3.11) and (3.12) therein we now have

$$
\begin{aligned}
\mathbf{h}^{(0)}= & z^{\prime} \mathbf{C}+\mathbf{D}, \\
\mathbf{h}^{(1)}= & i \alpha \sigma\left\{\frac{z^{\prime 2}}{2}\left[\mathbf{f}^{(0)}+\mathbf{D}\right]+\frac{z^{\prime 3}}{6} \mathbf{C}\right\}+z^{\prime} \mathbf{F}+\mathbf{G} \\
& -\frac{z^{\prime 2}}{2 \sigma}\left\{C_{x} \frac{\partial \sigma}{\partial x^{\prime}}+C_{y} \frac{\partial \sigma}{\partial y^{\prime}}\right\} \hat{\mathbf{k}},
\end{aligned}
$$

where $\mathbf{C}, \mathbf{D}, \mathbf{F}$ and $\mathbf{G}$ are vector-valued functions of $x^{\prime}$ and $y^{\prime}$ (whose components we denote by suffices) which are arbitrary at this stage, and $\hat{\mathbf{k}}$ is the unit vector in the $O z$ direction.

We again take the outer region to be that outside the sheet, and here we use the variables

$$
X=x^{\prime}=x / L, \quad Y=y^{\prime}=y / L, \quad Z=z / L, \quad \mathbf{H}^{*}=\mathbf{H} / \mathscr{H},
$$

and the asterisk will be dropped in the subsequent analysis. We replace $\mathbf{H}^{(p)}(X, Y, Z)$ by its Taylor expansion about $Z=0$, giving

$$
\mathbf{H}^{(p)}(X, Y, Z)=\mathbf{f}^{(0)}(X, Y)+Z \mathbf{f}^{(1)}(X, Y)+\cdots,
$$

where $\mathbf{f}^{(m)}(X, Y)=\frac{\partial^{m} \mathbf{H}^{(p)}}{\partial Z^{m}}(X, Y, 0)$, and again assume an expansion in the form

$$
\Phi=\Phi^{(0)}+\delta \Phi^{(1)}+\cdots
$$

Matching across the interfaces $\left(H_{x}, H_{y}, \frac{\partial H_{z}}{\partial Z}\right.$ and $B_{z}$ must be continuous), we obtain the boundary condition for the zeroth order terms as

$$
\frac{\partial^{2} \Phi_{+}^{(0)}}{\partial Z^{2}}=-\frac{\partial^{2} \Phi_{-}^{(0)}}{\partial Z^{2}}=-i \lambda \alpha \sigma\left(f_{z}^{(0)}-\frac{\partial \Phi_{+}^{(0)}}{\partial Z}\right)-\frac{1}{\sigma} \nabla_{2} \sigma \cdot \nabla_{2} \Phi_{+}^{(0)}
$$

where the suffixes \pm denote limiting values as the plane $Z=0$ is approached from above or below respectively, $\nabla_{2}$ denotes the vector operator $\left[\frac{\partial}{\partial X}, \frac{\partial}{\partial Y}\right], 1 / \lambda$ is the magnetic permeability nondimensionalized with respect to its value in free space. Equation (2.13) is the boundary condition obtained by Price [9], and it can be shown that $\Phi^{(0)}$ is an odd function in $Z$. For the first order terms, we again take

$$
\Phi^{(1)}=\phi^{(1 E)}+\phi^{(1 O)}
$$


where $\phi^{(1 E)}$ is an even function in $Z$ and satisfies the same boundary conditions as the corresponding function in Problem I, namely

$$
\frac{\partial \phi_{+}^{(1 E)}}{\partial Z}=\left(1-\frac{1}{\lambda}\right) f_{z}^{(1)}=-\frac{\partial \phi_{-}^{(1 E)}}{\partial Z},
$$

while $\phi^{(10)}$ is an odd function in $Z$ and satisfies the boundary condition

$$
\begin{gathered}
\frac{\partial^{2} \phi_{+}^{(1 O)}}{\partial Z^{2}}-i \lambda \alpha \sigma \frac{\partial \phi^{(1 O)}}{\partial Z}+\frac{1}{\sigma} \nabla_{2} \sigma \cdot \nabla_{2} \phi_{+}^{(1 O)}=-(\lambda-1)\left(f_{z}^{(2)}-\frac{\partial^{3} \Phi^{(0)}}{\partial Z^{3}}\right) \\
+i \alpha \sigma\left(\lambda-\frac{1}{3}\right) \frac{\partial^{2} \Phi_{+}^{(0)}}{\partial Z^{2}}+\frac{(\lambda-1)}{\sigma} \nabla_{2} \sigma \cdot \nabla_{2}\left(\frac{\partial \Phi^{(0)}}{\partial Z}-f_{z}^{(0)}\right) .
\end{gathered}
$$

Our problem is now the determination of three harmonic functions, $\Phi^{(0)}, \phi^{(1 E)}$ and $\phi^{(10)}$ satisfying the boundary conditions given by (2.13), (2.15) and (2.16) respectively. We note that, with a trivial rearrangement of terms, (2.13) and (2.16) are similar in form and hence, little extra effort is required to obtain the first order correction to the potential that is odd in $Z$ once a method of solution has been found for the zero order case.

\section{The integro-differential equations}

It is more convenient to work in terms of the integrated current density defined by

$$
\mathbf{I}=\int_{-1}^{\mathbf{l}} \mathbf{j} d z^{\prime}
$$

where $\mathbf{I}=\left[I_{x}, I_{y}, 0\right]$ and $\mathbf{I}=\mathbf{I}^{(0)}+\delta \mathbf{I}^{(1)}+\cdots$. The expressions for the zero and first order terms for $I$ as given by (4.8) and (4.9) in Problem I are still valid for the present case and are reproduced below.

$$
\mathbf{I}^{(0)}=2\left[\frac{\partial \Phi_{+}^{(0)}}{\partial Y},-\frac{\partial \Phi_{+}^{(0)}}{\partial X}, 0\right]
$$

and

$$
\mathbf{I}^{(1)}=2\left[\frac{\partial \chi}{\partial Y},-\frac{\partial \chi}{\partial X}, 0\right]
$$

where

$$
\chi=(1-\lambda)\left\{\frac{\partial \Phi_{+}^{(0)}}{\partial Z}-f_{z}^{(0)}\right\}+\phi_{+}^{(10)}
$$


Introducing the stream potentials $U(X, Y)$, and $V(X, Y)$ [12] satisfying

$$
\mathbf{I}^{(0)}=\nabla \times[0,0, U(X, Y)]
$$

and

$$
\mathbf{I}^{(1)}=\nabla \times[0,0, V(X, Y)] .
$$

Equations (3.2) and (3.5) imply

$$
\nabla \times \mathbf{I}^{(0)}=2 \frac{\partial^{2} \Phi_{+}^{(0)}}{\partial Z^{2}} \hat{\mathbf{k}}=-\nabla^{2} U \hat{\mathbf{k}}
$$

Equations (2.13), (3.5) and (3.7) now yield

$$
\nabla^{2} U-\frac{1}{\sigma} \nabla_{2} \sigma \cdot \nabla_{2} U=2 i \alpha \sigma \lambda\left(f_{z}^{(0)}-\frac{\partial \Phi_{+}^{(0)}}{\partial Z}\right) .
$$

It can be shown that any potential function $\phi$ may be given in terms of its distribution on the surface of the sheet [5, Section 6.093], and a little manipulation allows us to write

$$
\frac{\partial \phi}{\partial Z}(X, Y, Z)=-\iint_{S} \frac{\left(\mathbf{r}-\mathbf{r}^{\prime}\right) \cdot \nabla \phi}{2 \pi\left|\mathbf{r}-\mathbf{r}^{\prime}\right|^{3}} d S,
$$

where $S$ denotes the surface of the sheet and $\mathbf{r}$ and $\mathbf{r}^{\prime}$ are the position vectors of the observation and source points respectively. Hence, using this, (3.2) and (3.5), the integro-differential equation for $U$ is given by

$$
\nabla^{2} U-\frac{1}{\sigma} \nabla_{2} \sigma \cdot \nabla_{2} U=2 i \alpha \sigma \lambda\left(f_{z}^{(0)}+\iint_{S} \frac{\left(\mathbf{r}-\mathbf{r}^{\prime}\right) \cdot \nabla U}{4 \pi\left|\mathbf{r}-\mathbf{r}^{\prime}\right|^{3}} d S\right),
$$

and given the conductivity distribution, the stream potential can be calculated. The integro-differential equation is similar in form to that treated by the authors elsewhere $[10,11]$.

Repeating the analysis now for the first order potential $V$, (3.3) leads to

$$
\begin{aligned}
\left(\nabla \times \mathbf{I}^{(1)}\right) \cdot \hat{\mathbf{k}} & =2(\lambda-1)\left(f_{z}^{(2)}-\frac{\partial^{3} \Phi_{+}^{(0)}}{\partial Z^{3}}\right)+2 \frac{\partial^{2} \phi_{+}^{(1 O)}}{\partial Z^{2}} \\
& =-\nabla^{2} V .
\end{aligned}
$$

Use of (2.16) now gives

$$
\begin{aligned}
-\nabla^{2} V= & -\frac{2}{\sigma} \nabla_{2} \sigma \cdot \nabla_{2} \Phi_{+}^{(0)}+2 i \alpha \sigma \lambda \frac{\partial \phi_{+}^{(10)}}{\partial Z} \\
& -2 i \alpha \sigma\left(\frac{1}{3}-\lambda\right) \frac{\partial^{2} \Phi_{+}^{(0)}}{\partial Z^{2}}-\frac{2(\lambda-1)}{\sigma} \nabla_{2} \sigma \cdot \nabla_{2}\left(f_{2}^{(0)}-\frac{\partial \Phi_{+}^{(0)}}{\partial Z}\right)
\end{aligned}
$$


Using (3.3) to substitute for $\frac{\partial \phi_{+}^{(10)}}{\partial X}$ and $\frac{\partial \phi_{+}^{(10)}}{\partial Y}$ and then using (3.9) we obtain for $V$ the integro-differential equation

$$
\nabla^{2} V-\frac{1}{\sigma} \nabla_{2} \sigma \cdot \nabla_{2} V=2 i \alpha \sigma \lambda\left(g+\iint_{S} \frac{\left(\mathbf{r}-\mathbf{r}^{\prime}\right) \cdot \nabla V}{4 \pi\left|\mathbf{r}-\mathbf{r}^{\prime}\right|^{3}} d S\right),
$$

where

$$
g=\left(\frac{1}{3 \lambda}-1\right) \frac{\partial^{2} \Phi_{+}^{(0)}}{\partial Z^{2}}-\frac{(\lambda-1)}{2 \pi} \iint_{S} \frac{\left(\mathbf{r}-\mathbf{r}^{\prime}\right) \cdot \nabla\left(f_{z}^{(0)}-\frac{\partial \Phi_{+}^{(0)}}{\partial Z}\right)}{\left|\mathbf{r}-\mathbf{r}^{\prime}\right|^{3}} d S
$$

We see that (3.10) and (3.13) are similar so that the same method of solution can be employed for both the zero order and first order potentials that are odd functions of $z$.

\section{Solution methods}

Equation (2.15) and Gauss's Theorem show that $\phi^{(1 E)}$ is the potential of a distribution of magnetic poles on the plane $Z=0$ of strength $\frac{1}{2 \pi}(1-1 / \lambda) f_{z}^{(1)}$, so that

$$
\phi^{(1 E)}=-\frac{1}{2 \pi}\left(1-\frac{1}{\lambda}\right) \iint_{S} \frac{f_{z}^{(1)}\left(x^{\prime}, y^{\prime}\right)}{\sqrt{\left(x-x^{\prime}\right)^{2}+\left(y-y^{\prime}\right)^{2}}} d x^{\prime} d y^{\prime} .
$$

$\phi^{(1 E)}$ is also the magnetic response of the sheet to the primary field and is not due to the induced eddy current in the conducting sheet.

Unless the conductor contains a substantial proportion of ferro-magnetic material, its permeability is close to that of free-space [7, page 106]. We therefore take $\lambda=1$ so that $\phi^{(1 E)}$ is zero and only the first term of $g$ remains in (3.14). However, the solution for $\Phi^{(0)}$ and $\phi^{(10)}$ is still far from simple, the actual method to be used being influenced by the body shape and lateral extent of the sheet. Price [9] has considered the zeroth order solution in the time domain for infinite sheets and spherical shells for various conductivity distributions. When the sheet is of finite extent there is no analytic method that can handle both a prescribed conductivity profile and a finite geometry. Recourse has to be made to numerical techniques.

In our analysis here, we have tacitly assumed that the conductivity is representable by a nonzero differentiable function of position over the conducting sheet $S$ with derivatives of $\mathrm{O}(\Sigma / L)$. If the conductivity in $S$ tends to zero at the edge, a straight forward method would be to enclose the conductor by an arbitrary large rectangle over which a regular mesh may be imposed. On points of the rectangle outside $S$, both $U$ and $V$ satisfy Laplace's equation and it is easy to deduce that $U=0=V$ outside 
$S$. The same method would work if the surrounding medium has constant nonzero conductivity $\sigma_{0}$ say, provided that the conductivity distribution on $S$ is continuous across the edge. In this case the second term in each of (3.10) and (3.13) vanish and the integral equations will be different inside the arbitrary rectangle depending on whether the point is in $S$ or not. When the conductivity does not tend to that of the surrounding medium at the edge of the sheet, the above method will not work since the gradient of $\sigma$ will not be defined at the edge, thus violating one of the assumptions under which the perturbation scheme is derived. Moreover, spurious currents of potentially large magnitudes will be introduced wherever the discontinuity in $\sigma$ is large. In this case, it is necessary to solve for $U$ and $V$ over $S$ only. Lamontagne and West [6] did this for the zeroth order solution over a rectangular plate with constant conductivity and their results compared very well with those obtained from scale model experiments in air. Recently, Siew [10] used a numerical technique to solve (3.10) for the case of constant conductivity. In that paper, the surface of a plane sheet of arbitrary shape is first mapped onto a unit square. Thus, in the transformed $\xi \eta$-plane say, the sheet always occupies the region $0 \leq \xi \leq 1,0 \leq \eta \leq 1$. The numerically fitted set of curvilinear coordinates in the $X Y$-plane corresponds to a regular mesh in the $\xi \eta$-plane. Next, a cubature for $U$ is used inside the double integral which is then solved using a Gauss-Legendre scheme. Finite differences were used to approximate the Laplacian on the left hand side and the resulting system of equations was then solved by LU-factorization together with a sequence of mesh refinements. The scheme is readily adaptable to the case of variable conductivity. Provided the distribution $\sigma(X, Y)$ is known, the left hand side of (3.10) and (3.13) can still be replaced by a finite difference scheme. An advantage is that the same numerical procedure can be used for both the leading order and the first order equations (that is, for $U$ and $V$ ). We will not repeat the numerical details here, but the above method will be used to produce some numerical results for the case of variable conductivity.

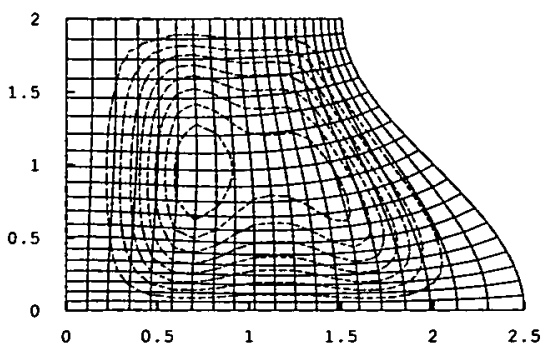

(a)

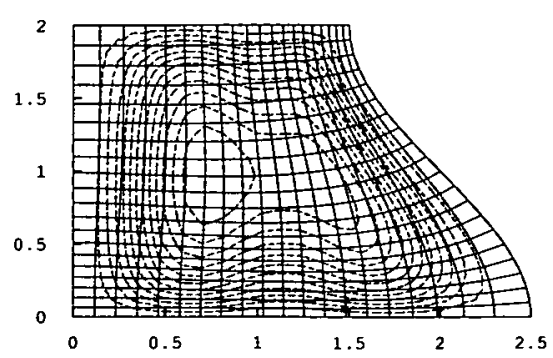

(b)

FIGURE 1. Stream potential maps for a uniform inducing field with conductivity given by (4.2). Zero contour on boundaries. Contours in steps of -0.004 . (a) Inphase component. (b) Quadrature component. 
Figure 1 shows a thin sheet whose right hand boundary is given by the curve with equation $x=2+0.5 \cos (\pi y / 2)$. This sheet is first mapped onto the unit square in the transform $\xi \eta$ space. For convenience, we have assumed a conductivity distribution given by

$$
\sigma(\xi, \eta)=1-0.9 \cos (4 \pi \xi) .
$$

Taking $\alpha=5$, the zeroth order stream potential maps due to a uniform primary magnetic field are shown. Using $\delta=0.1$, the first order correction leaves the potential maps qualitatively unchanged. Figure 2 shows the corresponding vertical components of the induced magnetic field along a line of traverse from the point $(-2,1,0.2)$ to $(4.5,1,0.2)$ in physical space. The horizontal scale $s$ measures distance from the initial point along the line of traverse, which passes over the sheet from $s=2$ to $s=4$. As expected, the measured field picks up the edges of the sheet quite clearly. On the other hand, the responses do not mirror the conductivity variation in the sheet.

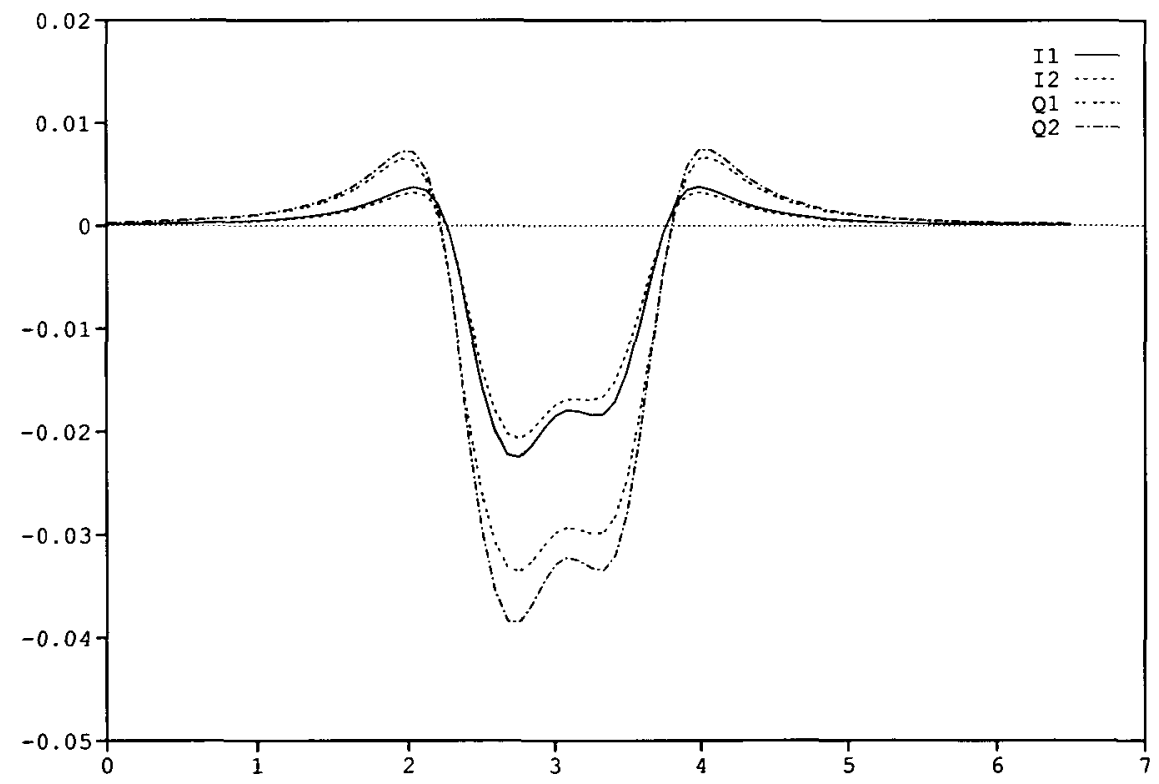

FigURE 2. Vertical components of the induced magnetic field due to a uniform primary field for the conductivity distribution given by (4.2). The horizontal scale $s$ measures distances along the path of traverse from $(-2,1,0.2)$ to $(4.5,1,0.2)$. The left hand edge of the sheet corresponds to $s=2$. \{I1,Q1\} and $\{12, Q 2\}$ denote the inphase and quadrature components correct to zero and first order respectively. 


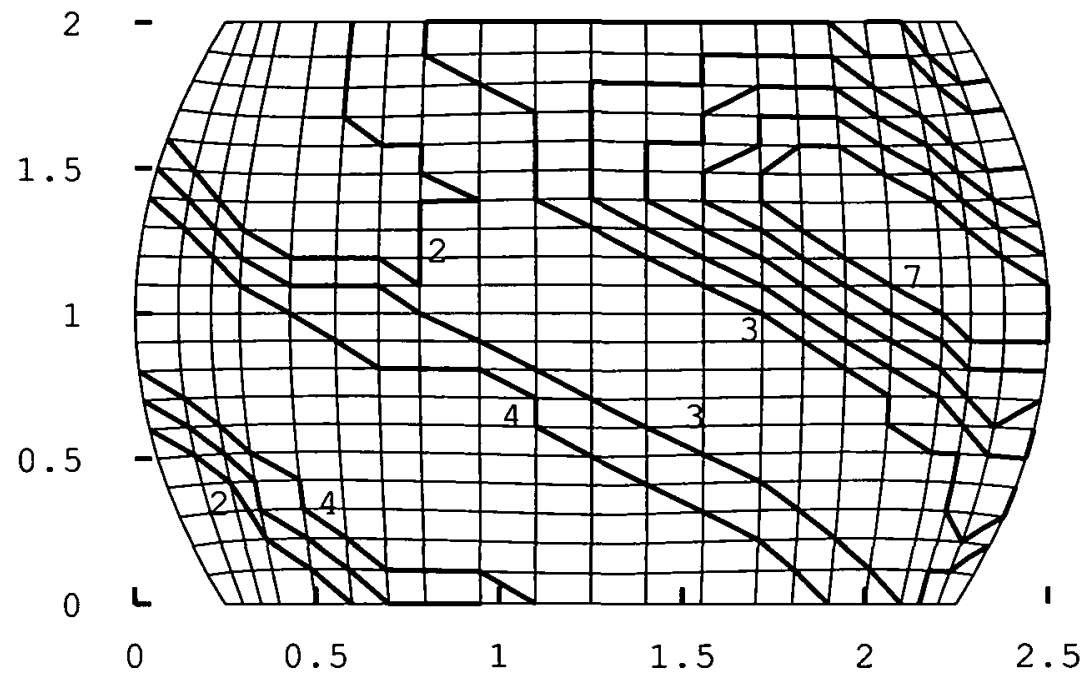

FIGURE 3. Conductivity contours.

In Figure 3, we consider a near rectangular shape with rounded ends where a conductivity distribution as shown is assumed. The actual contours are produced from prescribed values of the conductivity at the grid points of a $20 \times 20$ mesh covering the sheet. The inhomogeneity may be due to say, patches of clay in a lateritic deposit. Figure 4 gives the zeroth order stream potential maps, while Figure 5 gives the vertical components of the induced magnetic field. Here we have taken $\alpha=3$ and $\delta=0.1$.

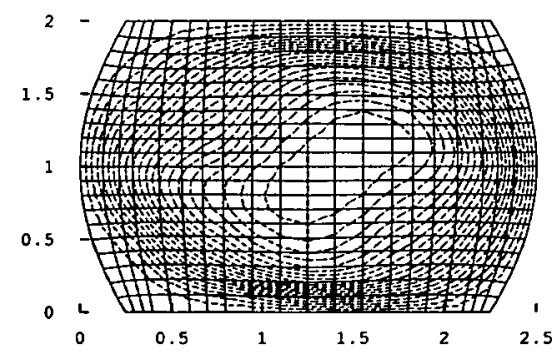

(a)

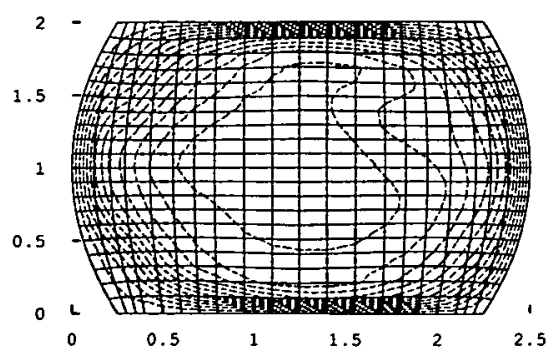

(b)

FIGURE 4. Stream potential maps for a uniform inducing field with conductivity distribution as given in Figure 3. Zero contour on boundaries. Contours in steps of -0.005 . (a) Inphase component. (b) Quadrature component. 


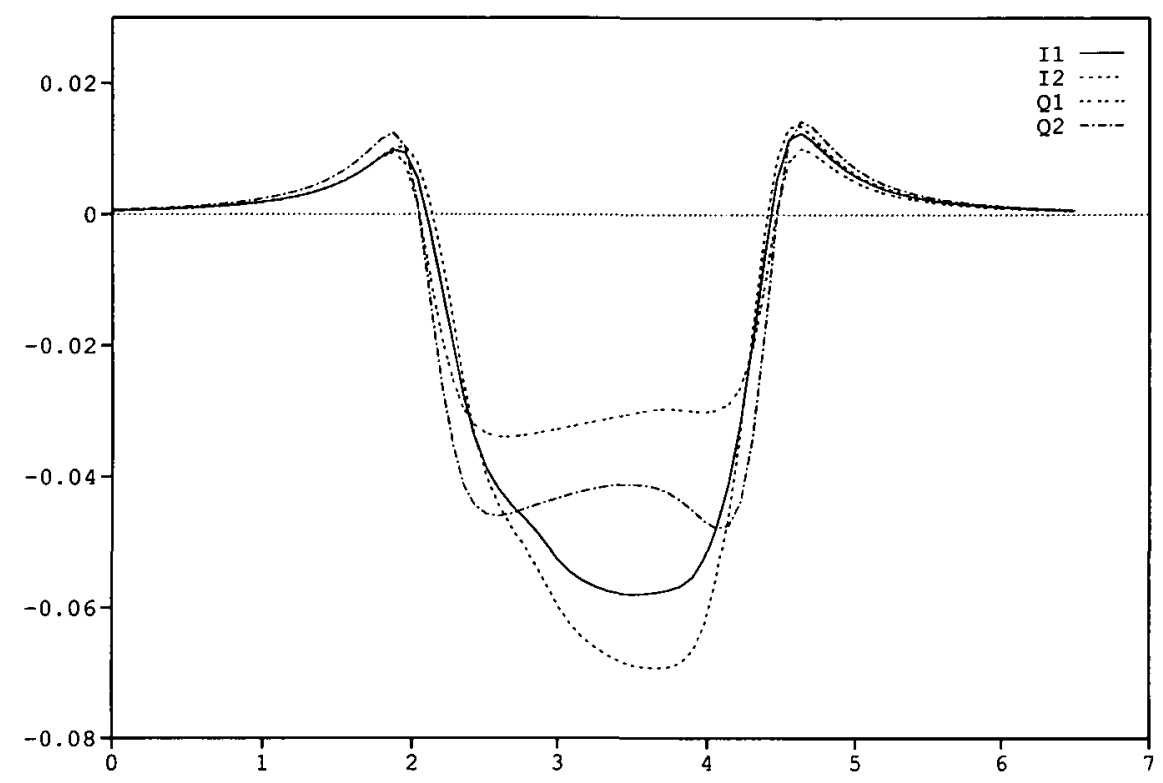

FIGURE 5. Vertical components of the induced magnetic field due to a uniform primary field for the conductivity distribution of Figure 3. The horizontal scale $s$ measures distances along the path of traverse from $(-2,1,0.2)$ to $(4.5,1,0.2)$. The response over the sheet corresponds to $2<s<4.5$. $\{11, \mathrm{Q} 1\}$ and $\{12, Q 2\}$ denote the inphase and quadrature components correct to zero and first order respectively.

A comparison of Figures 2 and 5 shows that there is little qualitative discrimination for the different conductivity contrast of the two examples used. A better picture of the variation in conductivity may be obtained by using an oscillating dipole source with a coincident receiving coil. The result is shown in Figure 6, where we have used the same traverse path over the second sheet. Now the inphase component of the induced field seems to pick up the variation in the conductivity quite well.

\section{Discussion}

We have presented a method whereby the electromagnetic responses due to bodies which are large in lateral extent compared to their thickness can be calculated numerically. The method allows us to compute the zeroth order as well as the first order correction efficiently for bodies with any prescribed conductivity contrast and a wide range of geometry. The peaks in the induced vertical magnetic field give a rough indication of the boundary of the body which is useful for the inverse prob- 


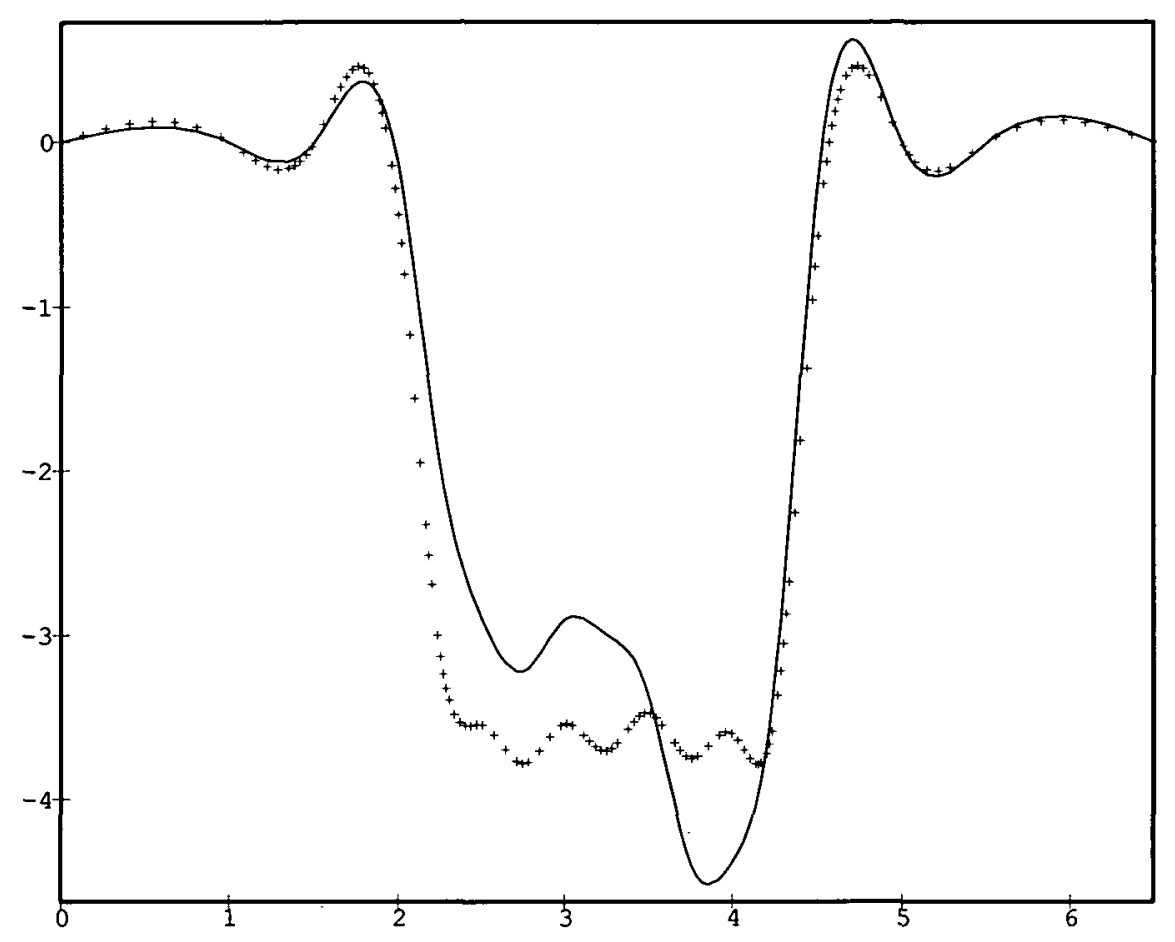

FIGURE 6. Vertical components of the leading induced magnetic field due to a coincident transmitting and receiving coil for the conductivity distribution of Figure 3. The horizontal scale $s$ measures distances along the path of traverse from $(-2,1,0.2)$ to $(4.5,1,0.2)$. The response over the sheet corresponds to $2<s<4.5$. - Inphase component. ++ Quadrature component.

lem. Conductivity contrasts in the sheet may be picked up qualitatively by use of a coincident transmitting and receiving coil to traverse the sheet. The result suggests that a possible application is in the area of the detection of flaws in bodies of wide lateral expanse. In the geophysical context we have assumed here that the body is surrounded by vacuum so that the method is valid when the surrounding medium is not magnetic or highly conducting.

\section{References}

[1] A. A. Ashour, "The induction of electric currents in a uniform circular disc", Q. J. Mech. Appl. Math. 3 (1950) 119-28. 
[2] A. A. Ashour and A. T. Price, "The induction of electric currents in a non-uniform ionosphere", Proc. R. Soc. Lond. A 195 (1948) 198-224.

[3] D. G. Hurley and P. F. Siew, "The decay of eddy currents in thin sheets and related water wave problems", IMA J. Appl. Math. 34 (1985) 1-21.

[4] D. G. Hurley and P. F. Siew, "A first-order correction to the theory for the electromagnetic response of a thin conducting sheet", Geophys. Prosp. 39 (1991) 527-41.

[5] H. Jeffreys and B. S. Jeffreys, Methods of mathematical physics, 3rd Ed. (Camb. Univ. Press, 1962).

[6] Y. Lamontague and G. F. West, "EM response of a rectangular thin plate", Geophysics 36 (1971) 1204-22.

[7] L. D. Landau and E. M. Lifshitz, Electrodynamics of continuous media, 2nd Ed. (Pergamon Press, Oxford, 1984).

[8] J. C. Maxwell, A Treatise on electricity and magnetism, Vol. 2, 3rd Ed. (Dover Publications Inc., 1891) 291-295.

[9] A. T. Price, "The induction of electric currents in non-uniform thin sheets and shells", Q.J.Mech. Appl. Math. 2 (1949) 282-310.

[10] P. F. Siew, "A numerical scheme for the electromagnetic response in thin conductors of arbitrary planar shape", J. Aust. Math. Soc. (B) 35 (1994) 479-497.

[11] P. F. Siew and D. G. Hurley, "Electromagnetic response of thin disks", J.Appl. Math.Phys. (ZAMP) 39 (1988) 619-33.

[12] W. R. Smythe, Static and dynamic electricity (McGraw Hill, London, 1968). 